\title{
ARG1 Gene
}

National Cancer Institute

\section{Source}

National Cancer Institute. ARG1 Gene. NCI Thesaurus. Code C103927.

This gene plays a role in the urea cycle. 